\title{
Global-Local Finite Element Analysis for Predicting Separation in Cord-Rubber Composites of Radial Truck Tires
}

\author{
Kyoung Moon Jeong*, Kee Woon Kim, Jang Hyeon Kim \\ R\&D Center, Kumho Tire Co. Inc., Yongin-si, Gyeonggi-do, South Korea \\ Email: ^kmjeong@kumhotire.com
}

How to cite this paper: Jeong, K.M., Kim, K.W. and Kim, J.H. (2019) Global-Local Finite Element Analysis for Predicting Separation in Cord-Rubber Composites of Radial Truck Tires. Open Journal of Modelling and Simulation, 7, 190-202.

https://doi.org/10.4236/ojmsi.2019.74011

Received: September 10, 2019

Accepted: October 20, 2019

Published: October 23, 2019

Copyright $\odot 2019$ by author(s) and Scientific Research Publishing Inc. This work is licensed under the Creative Commons Attribution International License (CC BY 4.0).

http://creativecommons.org/licenses/by/4.0/

\section{Open Access}

\begin{abstract}
A global-local finite element modeling technique is employed in this paper to predict the separation in steel cord-rubber composite materials of radial truck tires. The local model uses a finite element analysis in conjunction with a global-local technique in ABAQUS. A 3-dimensional finite element local model calculates the maximum cyclic shear strain of an interface between steel cord and rubber materials at the carcass ply shoulder region. It is found that the maximum cyclic shear strain is reliable as a result of the analysis of carcass ply separation in radial truck tires. Using the analysis of the local model, a study of the cyclic shear strain is performed in the shoulder region and used to determine the carcass ply separation. The effect of the change of carcass ply design on the separation in steel cord-rubber composite materials of radial truck tires is discussed.
\end{abstract}

\section{Keywords}

Global-Local Finite Element Analysis, Separation, Composite Material, Truck Tire

\section{Introduction}

In recent years, composite materials have been widely used in several applications due to their superior mechanical properties including high strength, high stiffness, and low density. In the past, the remarkable advancements in theoretical and computational methods for analyzing composite materials have been made. However, until recently, investigating the effect of lamina properties and lay-up configurations on the strength of composites is an active field of research. Finite element method (FEM), extended finite element method (XFEM), and 
global-local finite element method (GLFEM) are powerful tools for solving the boundary value problems. Tire durability due to large local loading, stiffness discontinuities, and production flaws is frequently related to the fracture of tire components such as belt separation, carcass ply separation, or lug cracking in radial truck tire [1] [2] [3] [4] [5]. Influence factors on fracture and durability of rubber material and tires are manifold. Accordingly, understanding the fracture and fatigue behaviors of the tire component is the key to improve durability and assess lifetime of the radial truck tire [6].

A separation between the plies in a multi-ply radial passenger car tire is a rare event. It is most closely associated with bias and bias-belted tires. Even in radial passenger car tires with ply angles slightly off radial, a separation between the plies is rare. These separations will appear as bulges or softness in sidewall. Unless they have cracked open to allow a good inspection, cutting open the sidewall is the only method to inspect the interior sections [7]. Unlike passenger car tires, carcass plies are made of steel material in radial truck tires. Usually an externally visible bulge on the top sidewall of the truck tire indicates that cord-rubber composites have been separated inside the carcass ply shoulder region and is the risk of truck tire failure at some time in the future. The separation example from a cord-rubber composite material in truck tires is as shown in Figure 1. Figure 1(a) and Figure 1(b) show the outside and inside surface of the crack propagation at the carcass ply shoulder region. It is shown that the cord and rubber are completely separated from the cut section surface of the truck tire as shown in Figure 1(c). In general, the separation in carcass ply of radial truck tires first begins to rust on the surface of the steel cord and then completely separate as the crack grows around the steel cord as shown in Figure 2.

Due to the combination of high stress/strain, high temperature and large thickness, the tire shoulder and bead parts are among the most vulnerable areas in tire components. As for the radial truck tires, the combination of high load and high inflation pressure makes the belt edge or the ply turn-up endurance problem more severe. Several researchers have studied fracture mechanics of tire. Fracture mechanics provides a fundamental approach to analyze the fracture and fatigue crack growth in tires. Ebbott [1] used a finite element-based

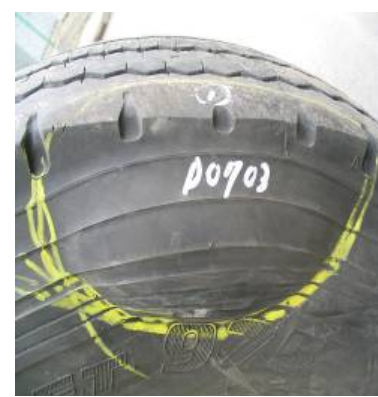

(a)

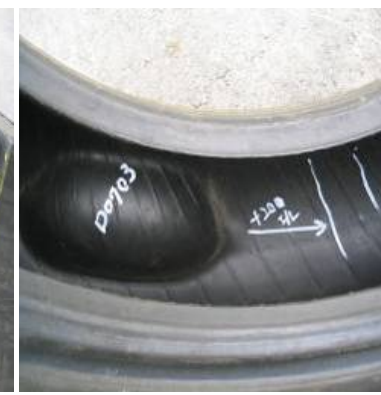

(b)

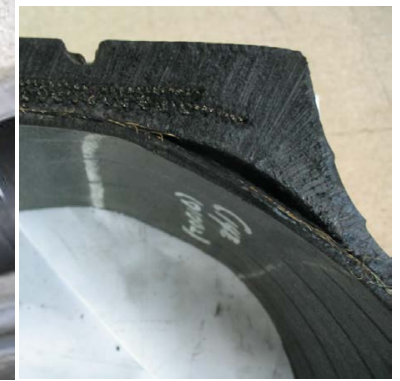

(c)

Figure 1. Separation example from a cord-rubber composite tire. (a) Outside surface; (b) inside surface; (c) cut section. 


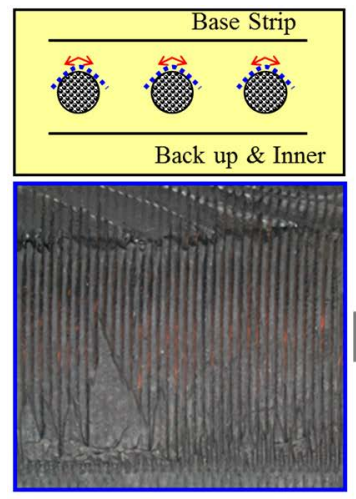

(a)

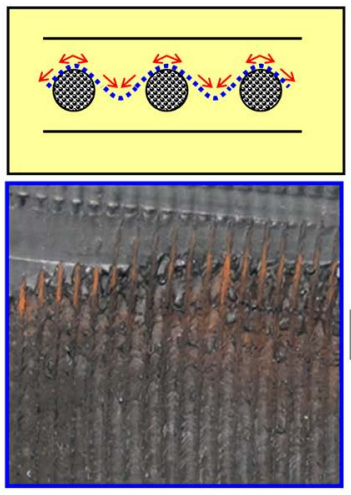

(b)

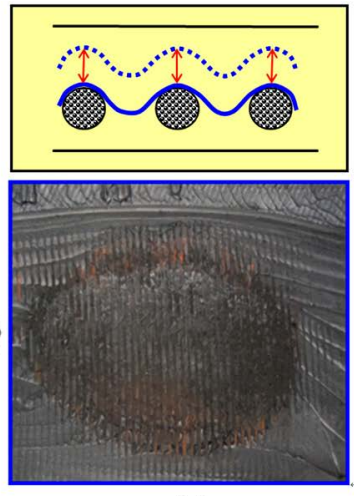

(c)

Figure 2. The process of carcass ply separation of a radial truck tire. (a) Threshold; (b) crack growth; (c) failure.

method to analyze the severity of internal cracks in cord-rubber structures. Yan et al. [8] have studied for the endurance of radial truck tires with finite element modeling by using two approaches: stress analysis parameter approach and tire structure parameter approach. Han et al. [3] and Jeong et al. [4] performed a failure analysis of truck tires based on fracture mechanics, using a global-local finite element model. The suggested model has proved effective in a variety of analysis for the prediction of tire lifetime. Zhong [9] formulated to study fatigue crack growth and durability in tires using a three-dimensional fracture mechanics model. The application of this model in a radial truck tire reveals fracture characteristics of belt edge cracks and helps to explain mechanical and material changes in the tire subject to indoor accelerated durability tests. However, the carcass ply separation of radial truck tires has not been studied.

The main purpose of this paper is to predict the separation in cord-rubber composite materials of radial truck tires based on static and steady-state rolling assumption. Cord-rubber composites primarily control the overall performance characteristics of tires. Micromechanical parameters including fiber and void volume fractions cannot be studied by the macroscopic modeling of carcass in tires. In order to predict the separation of carcass ply in truck tires, the local model uses a finite element analysis in conjunction with a global-local finite element analysis in ABAQUS [10]. It is found that the maximum cyclic shear strain is reliable as a result of the analysis of carcass ply separation in radial truck tires. Using the analysis of the local model, a study of the shear strain is performed in the shoulder region and used to determine the separation in carcass ply shoulder region of radial truck tires. The effect of the design change on the separation in steel cord-rubber composite materials of radial truck tires is discussed.

\section{Finite Element Modeling}

\subsection{Tire Model}

A tire usually consists of several rubber components, each of which is designed 
to contribute to some particular factors for tire performance in addition to several cords and rubber composites. These components play a role in maintaining the stiffness and strength required in a tire. Figure 3 shows the 2D and 3D finite element mesh on the general structure with a size of 12R22.5 found in radial truck and bus tires, where the roles of tire components are well described in a book by Clark [11]. It consists of a carcass ply, four belt plies, steel chafer, bead wires and several rubber components. Figure 3(a) shows a two-dimensional finite element model generated by solid-modeling program, in which 4-node quadrilateral and 3-node triangular elements are used. The global three-dimensional finite element model as shown in Figure $3(\mathrm{~b})$ is generated by rotating the two-dimensional cross section of a finite element through a 360 degree and is defined the unequal angular increments. The global model contains very small segments (2.0 degree) at the contact part as shown in Figure 3(b).

\subsection{Material Model}

In general, the material composition of a radial truck tire is largely divided into fiber-reinforced rubber parts and the remaining pure rubber part. The fiber-reinforced rubber parts are composed of a single-ply steel carcass, four steel belt layers, and several steel bead cords. Because the structures of fiber-reinforced rubber parts are very complex, their material models are chosen based on the goal of the numerical simulation. When the fiber-reinforced rubber parts are modeled using finite element analysis, the physical properties of the rubber and cords are combined to yield the resulting physical behavior of the FRR [12] [13], the internal cords are modelled using beam elements [14] or using rebar elements [15] [16] [17] [18]. Studies that have modeled belts based on a single physical behavior have de-fined the behavior using isotropic elasticity [12], hyperelasticity [19], the Halpin-Tsai composite equation [11], or theoretically derived belt constitutive equations [20] [21].

Rubbers except for the FRR parts are modeled by the penalized first-order Mooney-Rivlin model in which the strain-energy density function is defined [13].

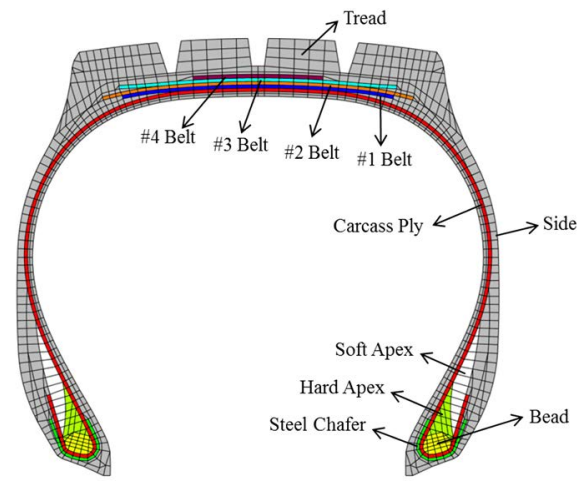

(a)

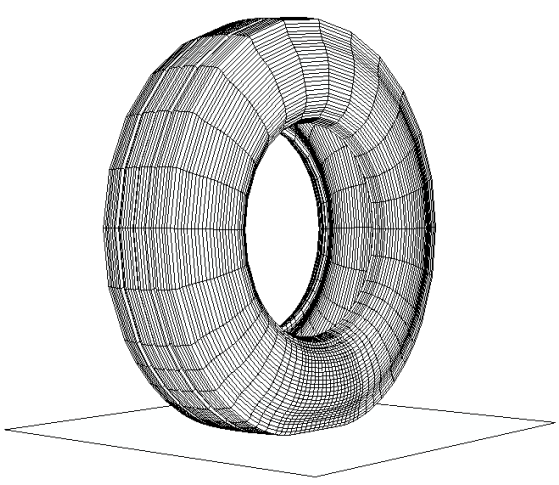

(b)

Figure 3. Finite element modeling of 12R22.5 tire. (a) 2-D section mesh; (b) 3-D model. 


$$
W\left(J_{1}, J_{2}, J_{3} ; K\right)=C_{10}\left(J_{1}-3\right)+C_{01}\left(J_{2}-3\right)+\frac{1}{K}\left(J_{3}-1\right)^{2}
$$

where $J_{i}$ are the invariants of the Green-Lagrangian strain tensor and $C_{10}$ and $C_{01}$ are the rubber material constants determined from the experiment. On the other hand, $K$ is a sort of penalty parameter controlling the rubber incompressibility. The shear modulus $\tau$ and the bulk modulus $\kappa$ of rubber are related as $2\left(C_{10}+C_{01}\right)=\tau$ and $K=2 \kappa$, from which one can easily obtain the following relation for Poisson's relation:

$$
v=\left[3 K / 4\left(C_{10}+C_{01}\right)-2\right] /\left[3 K / 2\left(C_{10}+C_{01}\right)+2\right] .
$$

It is clear that the incompressibility of the rubber is applied asymptotically as the penalty parameter approaches infinity, however, in general, it is better to choose 100 near $K$ for a stable transient dynamic response with the reasonable time step size.

\subsection{Composite Model}

Structural tire analysis is often performed using the cured tire geometry as the reference configuration for the finite element model. However, the cord geometry is more conveniently specified with respect to the "green" or uncured, tire configuration. The tire lift equation provides mapping from the uncured geometry to the cured geometry as shown in Figure 4. This study can specify the spacing and orientation of the rebar cords with respect to the uncured configuration and let Abaqus [10] map these properties to the reference configuration of the cured tire. Using a cylindrical coordinate system, the spacing, $s$, and angular orientation, $\alpha$, in the cured tire are obtained from

$$
\sin \alpha=\frac{r \sin \alpha_{0}}{r_{0}(1+e)}, \quad s=s_{0} \frac{r \cos \alpha}{r_{0} \cos \alpha_{0}},
$$

where $r$ is the position of the rebar along the radial direction in the cured geometry, $r_{0}$ is the position of the rebar in the uncured geometry, $s_{0}$ is the spacing in the uncured geometry, $\alpha_{0}$ is the angle measured with respect to the projected local 1-direction in the uncured geometry, and $e$ is the cord extension ratio. A local cylindrical coordinate system must be defined for the rebar if the rebar is associated with three-dimensional elements.

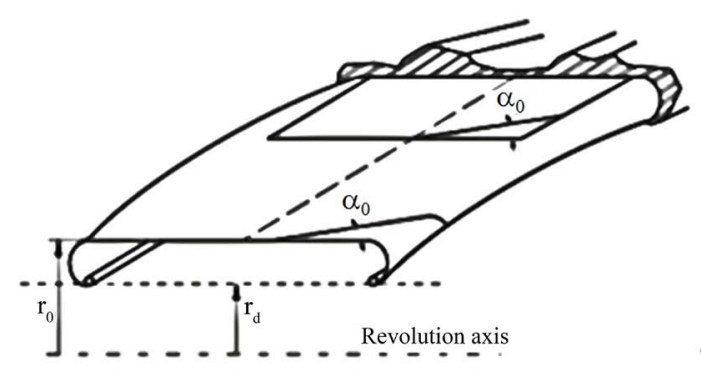

(a)

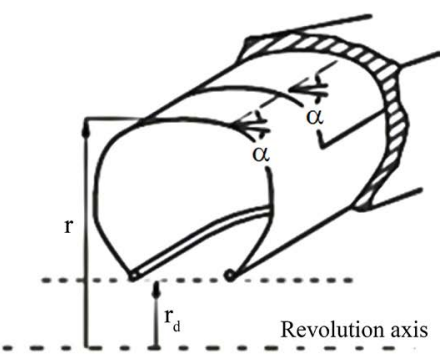

(b)

Figure 4. Mapping between uncured and cured tire rebar geometry [10]. (a) Uncured geometry; (b) cured geometry. 


\subsection{Local Model}

The carcass ply topping rubber is the rubber coating that encapsulates the radial ply reinforcing cords. The topping rubber is calendered onto the carcass ply cords in thin sheets. It is analyzed that the reason is that the carcass ply provides the strength to contain the air pressure and provide for sidewall impact resistance. There are many locations in a tire where a crack might exist. However, cracks at limited locations play dominant roles in the tire failure process. The critical locations, such as belt edge, carcass ply and ply turn-up, can be determined either from experience, such as observations of tire failures in the field or in lab tests or from finite element analysis of tires. Regions with local deformation, stress concentrations or abrupt changes in material stiffness, such as the interface between steel cord and rubber, are the locations where cracks are most likely to occur. In tire finite element modeling, the critical locations can be selected based on maximum cyclic strain energy density or maximum shear strain depending on material properties and local geometry. The local model is made of the back-up liner, carcass cord and topping rubber, and base strip and has a very small dimension to be embedded into the carcass ply shoulder region as shown in Figure 5.

\subsection{Boundary Condition}

To simulate the contact between the tire and the rim, the general purpose interface is defined between a tire slave surface and a rigid body surface of the rim. The inner and outer rim profiles are both modeled by axisymmetric rigid surfaces. Contact with friction is considered between the tire and the rim. The coefficient of friction is assumed to be 0.3 . No other interface is used to model the contact between the rubber matrix and the beads or between the rubber matrix and the different layers, such as carcass ply, belt, bead wrap and steel chafer.

\section{Result and Discussions}

\subsection{Global-Local Finite Element Analysis}

A global-local technique can be applied quite generally in finite element analysis. The material response defined for the local model may be different from that

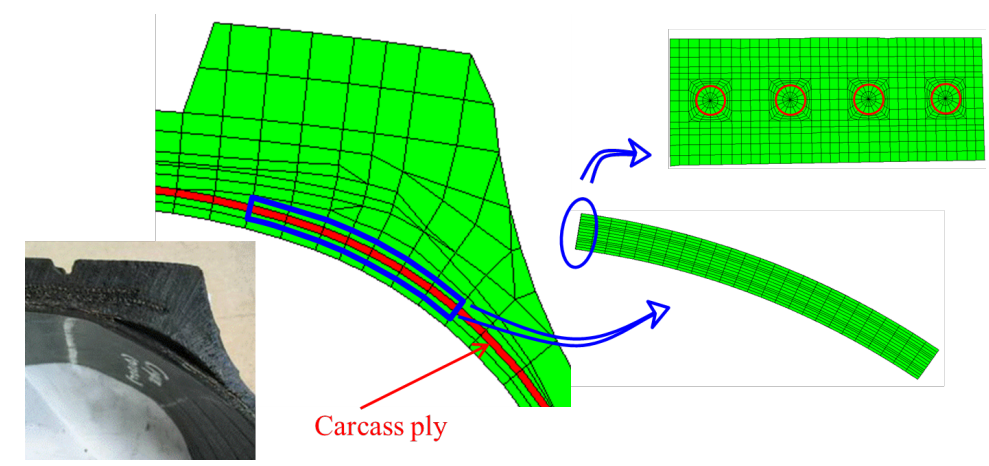

Figure 5. The process of local finite element modeling at the carcass ply shoulder region. 
defined for the global model. Both the global model and local model can have nonlinear response. The global-local technique is used to study a local part of a model with a refined mesh based on interpolation of the solution from an initial, relatively coarse, global model and is most useful when it is necessary to obtain an accurate, detailed solution in a local region and the detailed modeling of that local part region has negligible effect on the overall solution. The model whose solution is interpolated onto the relevant parts of the boundary of the local model is referred to as the global model. Driven variables are defined as those variables in the local model that are constrained to match results from the global model. In this paper, driven variables can be degrees of freedom at nodes in the node-based technique [10].

Using the global-local finite element method outlined in the previous section, a comprehensive study of a radial truck tire with a carcass ply separation is conducted. The local model is formed using a displacement boundary condition in a global finite element analysis of radial truck tire. Figure 6 is the procedure of a global-local finite element analysis for predicting separation of cord-rubber composite materials of radial truck tires.

\subsection{Separation Analysis of Carcass Ply}

In order to obtain the results of global-local finite element analysis, a radial truck tire, 12R22.5, is selected as the design change. It is decided to vary the carcass ply gage or carcass ply line, which have a significant impact on the carcass ply separation. The design change in the carcass ply shoulder region in the radial truck tires with the different carcass ply gage or carcass profile are shown in Figure 7. Figure 7 (a) shows a reference construction. In Figure $7(\mathrm{~b})$, the carcass cord diameter is the same as version 1, but the topping rubber is about $0.8 \mathrm{~mm}$ larger than version 1. Figure 7 (c) shows versions 3 and 4 with the profile change of carcass ply. Because the test verification of the simulation results of the carcass ply separation is very difficult, the design version is selected to obtain reasonable results using the simulation results of carcass ply separation according to the design change.

Using the local structure of Figure 7(a), the simulation is performed according to the inflation pressure and vertical loading change. The strain energy, stress,

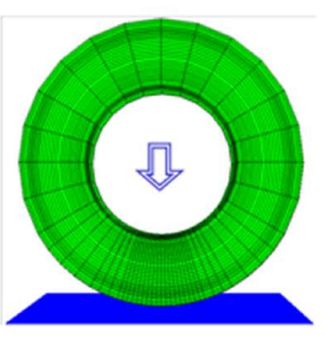

(a)

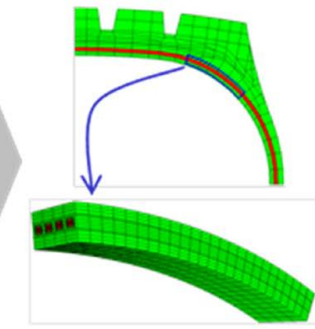

(b)

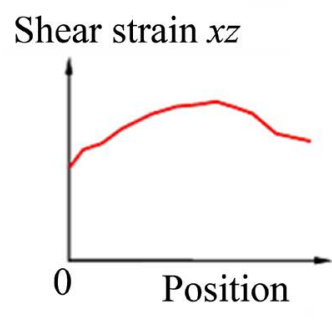

(c)

Figure 6. The procedure of a global-local finite element analysis. (a) Global model; (b) local model; (c) results. 


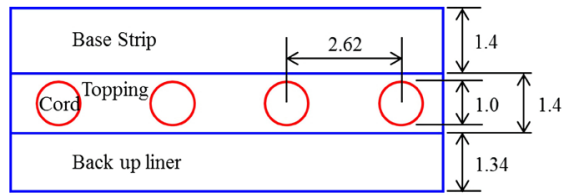

(a)

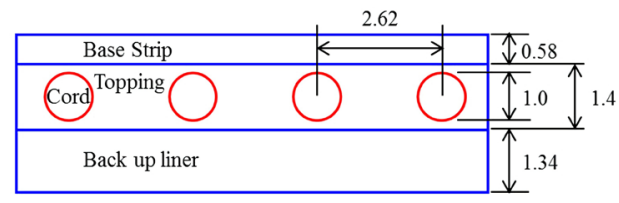

(c)

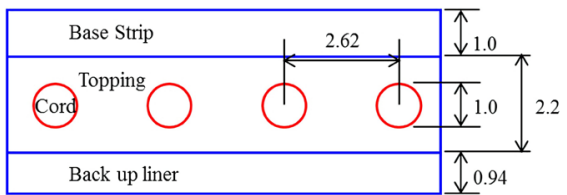

(b)

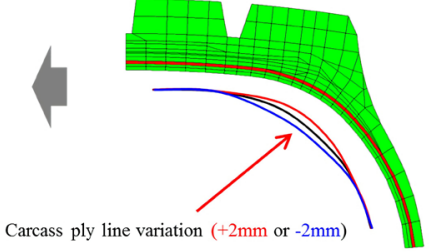

(d)

Figure 7. Versions with different designs in the carcass ply shoulder region. (a) Version 1; (b) Version 2; (c) Versions 3 \& 4; (d) carcass ply line variation.

strain, and temperature distribution were analyzed to select the most suitable simulation results for carcass ply separation. It is found that the cyclic shear strain increased with decreasing inflation pressure or increasing vertical loading as shown in Figure 8 and Figure 9. The effect of the vertical loading on the carcass ply separation is greater than the inflation pressure. Therefore, it can be seen that is important for the driver to maintain a proper load in order to prevent carcass ply separation in radial truck tires. It is found that the cyclic shear strain $X Z$ is reliable as a result of the analysis of carcass ply separation in radial truck tires.

The simulation results of carcass ply separation with the design change are shown in Figure 10 and Figure 11. The version 3 is vulnerable to carcass ply separation, and version 4 is the best as shown in Figure 10. It is found that the carcass ply separation is significantly affected by carcass profile changes compared to topping rubber gage change. In the local model, the measurement position of the maximum shear strain coincides with the crack initiation position of the carcass ply separation as shown in Figure 11. This result agrees with the experimental observation in Figure 1(c). Using the global-local finite element analysis proposed in this paper, we can confirm that the prediction of carcass ply separation in radial truck tires is valid.

\subsection{Steady-State Rolling Analysis}

The steady-state rolling contact analysis permits a local fine mesh near the contact zone while the transient rolling contact analysis requires a fine mesh along the entire tire surface. It leads to a large reduction of computation time in the steady-state rolling analysis. It is assumed that vehicle travels at $80 \mathrm{~km} / \mathrm{h}$ for classification of an angular velocity of a tire. Driving conditions considered in this paper are free-rolling, traction, and braking in order to understand how different driving contribute to the detrimental effects of carcass ply separation in truck tire failure. Figure 12 shows the cyclic shear strain for the driving condition. It is 


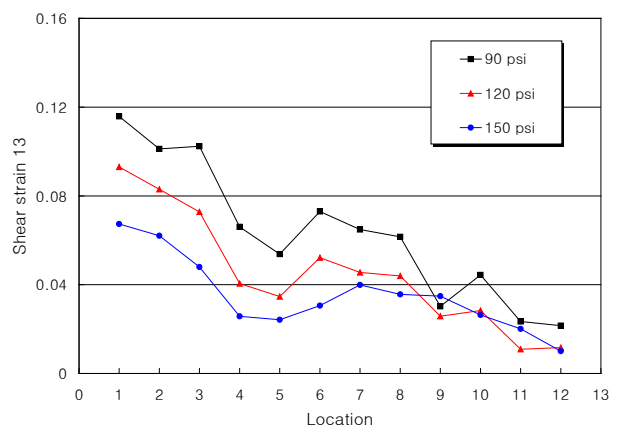

(a)

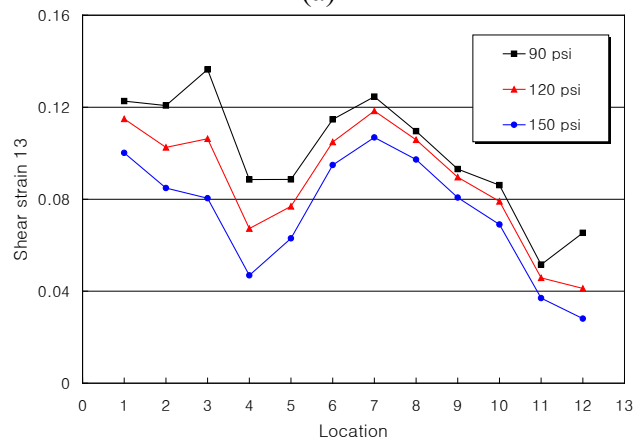

(b)

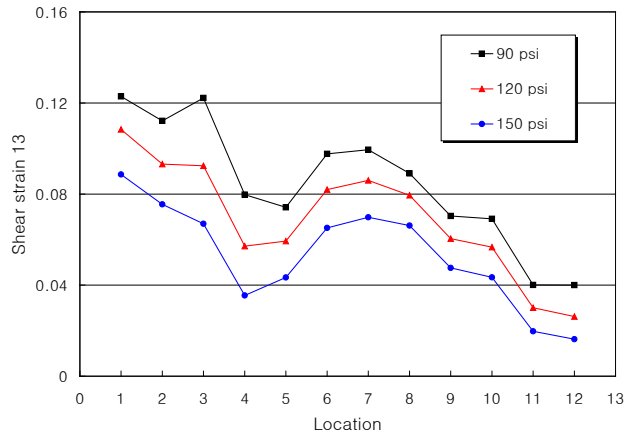

(b)

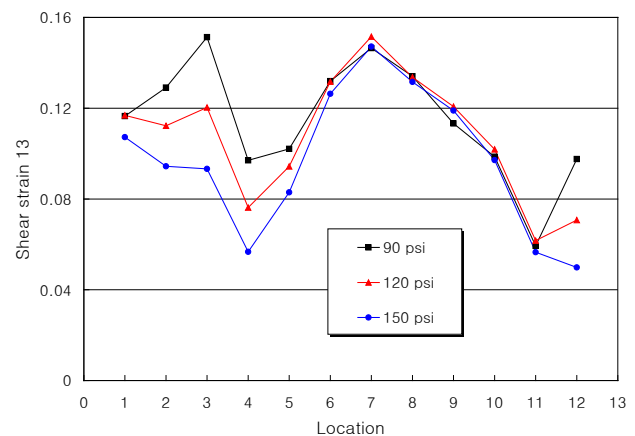

(d)

Figure 8. The effect of inflation pressure on the cyclic shear strain. (a) $2345 \mathrm{kgf}$ (70\%); (b) $3350 \mathrm{kgf}$ (100\%); (c) 4355 kgf (130\%); (d) 5695 kgf (170\%).

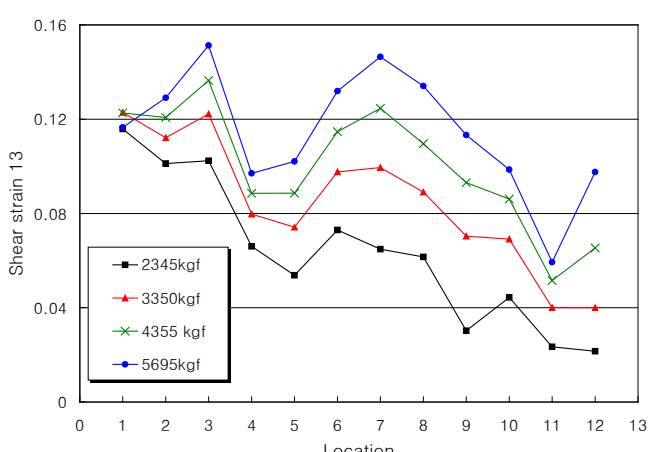

(a)

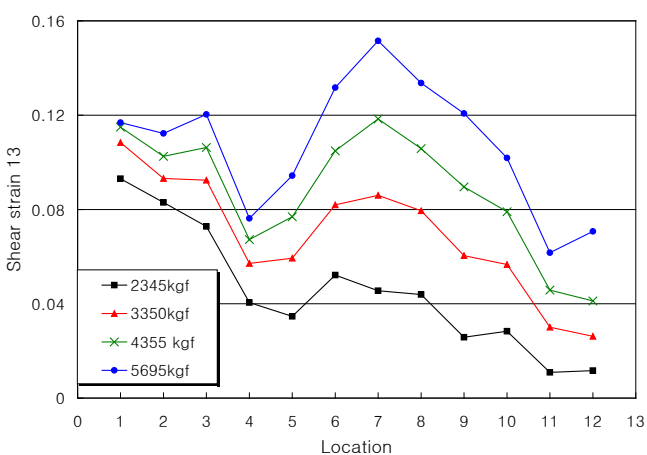

(b)

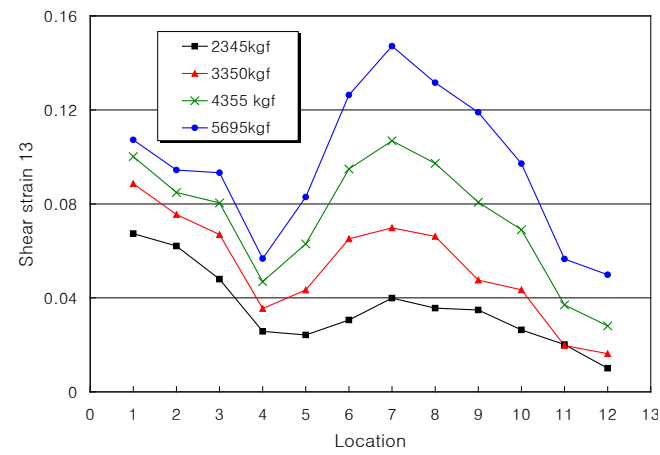

(c)

Figure 9. The effect of vertical loading on the cyclic shear strain. (a) 90 psi (75\%); (b) 120 psi (100\%); (c) 150 psi (125\%). 


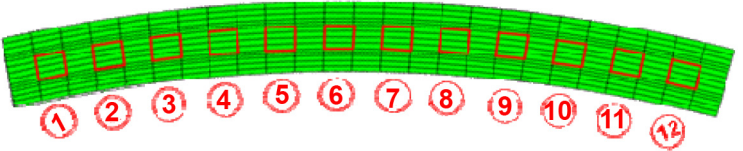

(a)

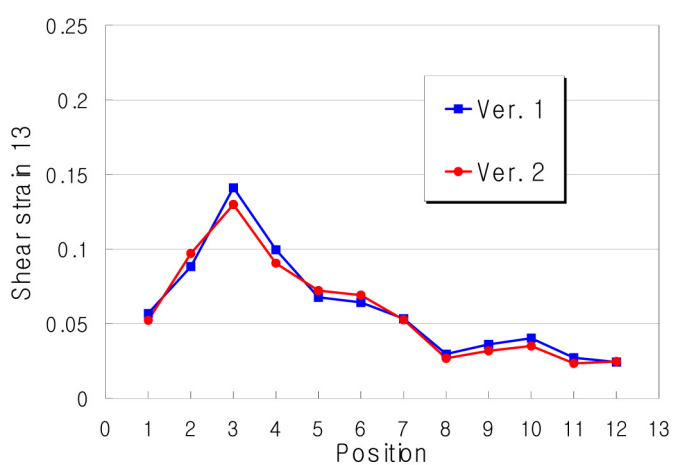

(b)

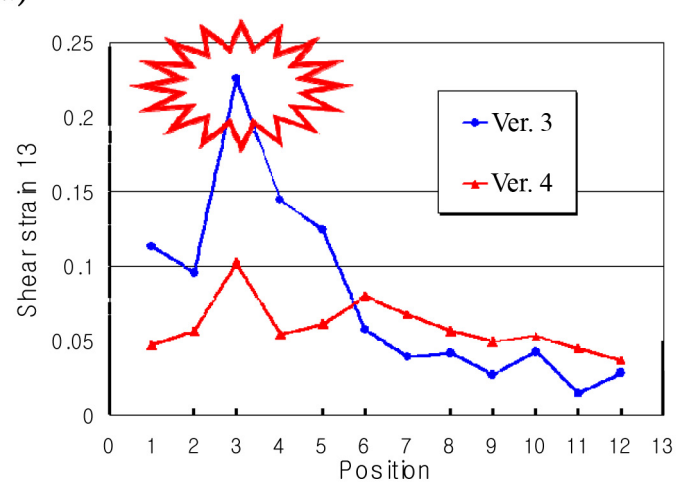

(c)

Figure 10. Cyclic shear strain $13(x z)$ at the carcass ply shoulder region. (a) Measured position of local model; (b) the effect of topping rubber gage; (c) the effect of carcass ply profile.

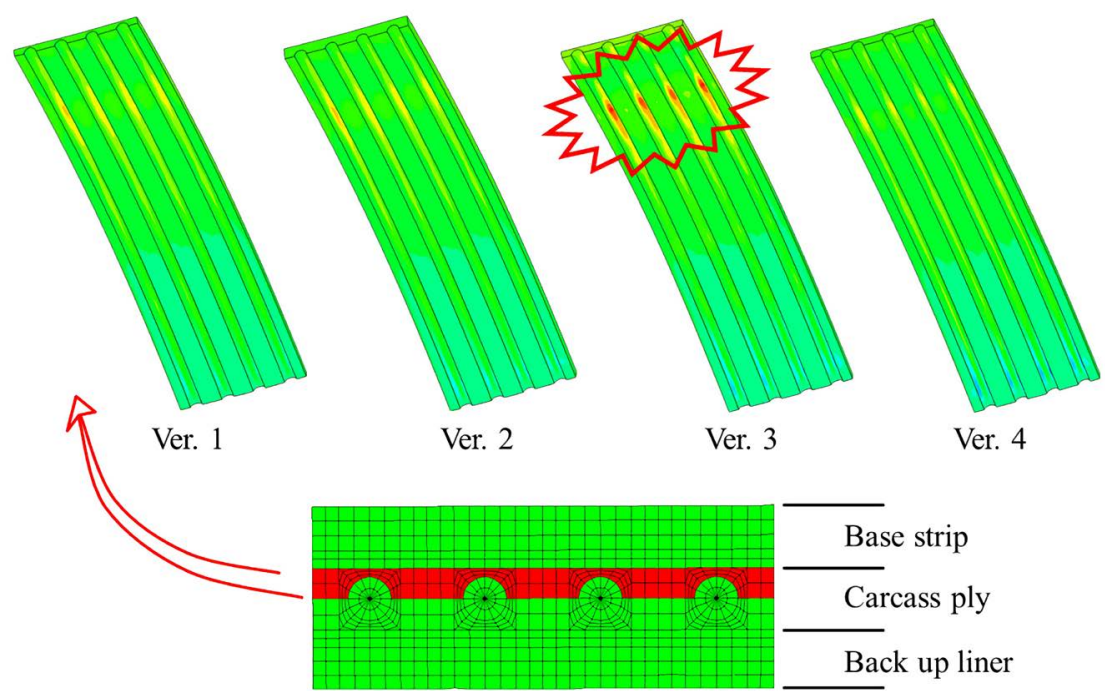

Figure 11. Cyclic shear strain $x Z$ component along the cord-rubber interface.

found that the maximum cyclic shear strain is not affected by the driving conditions.

\section{Conclusion}

The global-local finite element modeling technique is employed to predict the separation in cord-rubber composite materials of radial truck tires based on the static loading analysis and the steady-state rolling analysis. The carcass ply separation in radial truck tires begins to rust on the surface of the steel cord and then completely separate as the crack grows around the steel cord. In order to study the carcass ply separation, the global-local finite element analysis is used. It is 


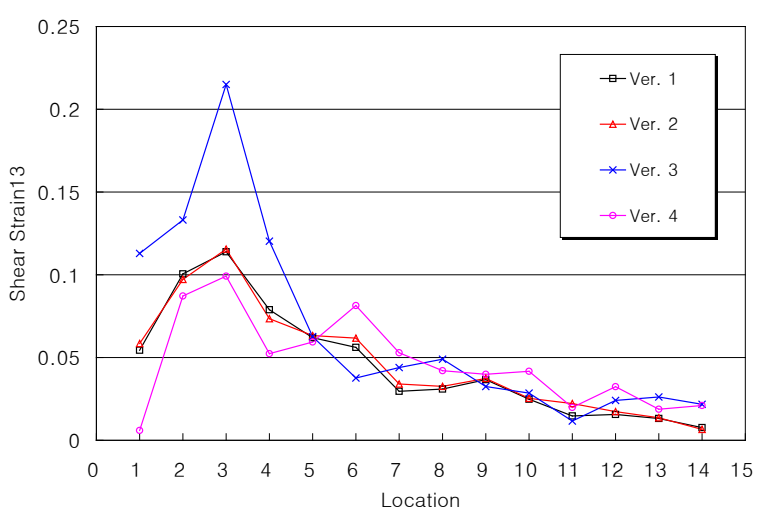

(a)



(b)

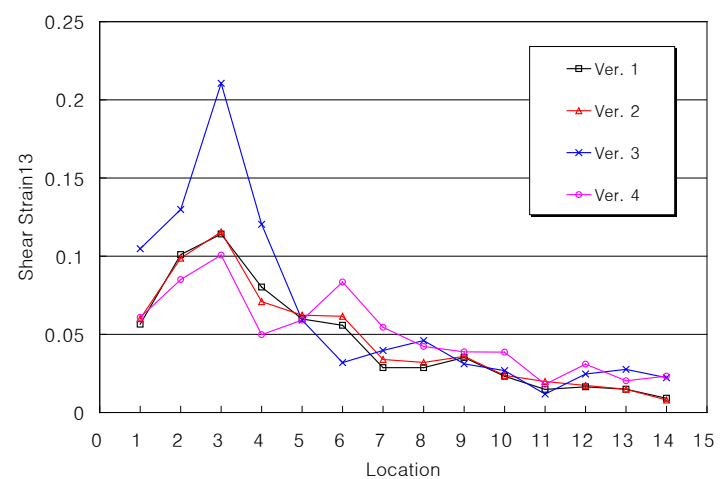

(c)

Figure 12. The cyclic shear strain with respect to driving conditions. (a) Free-rolling status; (b) traction status; (c) braking status.

found that the maximum cyclic shear strain can be used to determine which designs are more likely to cause carcass ply separation. The effect of the change of carcass ply design on the separation in steel cord-rubber composite materials of radial truck tires is discussed. It is found that the prediction of carcass ply separation using a global-local finite element method will be useful for the improvement of the separation of radial truck tire.

\section{Acknowledgements}

The present study was supported by the Center for Environmentally Friendly Vehicles (CEFV) under the project "development of the global top eco-friendly tire for reduction of tire wear particles and carbon dioxide" through the Ministry of Environment (ME, Republic of Korea).

\section{Conflicts of Interest}

The authors declare no conflicts of interest regarding the publication of this paper.

\section{References}

[1] Ebbott, T.G. (1996) An Application of Finite Element Based Fracture Mechanics 
Analysis to Cord-Rubber Structure. Tire Science and Technology, 24, 220-235. https://doi.org/10.2346/1.2137520

[2] Mars, W.V. (2001) Multiaxial Fatigue Crack Initiation in Rubber. Tire Science and Technology, 29, 171-185. https://doi.org/10.2346/1.2135237

[3] Han, Y.H., Becker, E.B., Fahrenthold, E.P. and Kim, D.M. (2004) Fatigue Life Prediction for Cord-Rubber Composite Tires Using a Global-Local Finite Element Method. Tire Science and Technology, 32, 23-40. https://doi.org/10.2346/1.2186772

[4] Jeong, K.M., Beom, H.G., Kim, K.W. and Cho, J.R. (2011) Fatigue Life Prediction for Radial Truck Tires Using a Global-Local Finite Element Method. Interaction and Multiscale Mechanics, 4, 35-47. https://doi.org/10.12989/imm.2011.4.1.035

[5] Jeong, K.M. (2016) Prediction of Burst Pressure of a Radial Truck Tire Using Finite Element Analysis. World Journal of Engineering and Technology, 4, 228-237. https://doi.org/10.4236/wjet.2016.42022

[6] Govindjee, S. (2001) Firestone Tire Failure Analysis. http://faculty.ce.berkeley.edu/sanjay/REPORT_Secure.PDF

[7] Giapponi, T.R. (2008) Tire Forensic Investigation: Analyzing Tire Failure. SAE International, 117-118.

[8] Yan, X., Wang, Y. and Feng, X. (2002) Study for the Endurance of Radial Truck Tires with Finite Element Modeling. Mathematics and Computers in Simulation, 59, 471-488. https://doi.org/10.1016/S0378-4754(01)00429-3

[9] Zhong, X.A. (2006) Computational Fracture Mechanics Analysis of Truck Tire Durability. Journal of Applied Mechanics, 73, 799-806. https://doi.org/10.1115/1.2069983

[10] ABAQUS (2014) Analysis User's Manual, Version 6.14. Dassault Systemes Simulia, Inc.

[11] Clark, S.K. (1982) Mechanics of Pneumatic Tires. US Department of Transportation, National Highway Traffic Safety Administration.

[12] Li, Y., Liu, W.Y., and Frimpong, S. (2012) Effect of Ambient Temperature on Stress, Deformation and Temperature of Dump Truck Tire. Engineering Failure Analysis, 23, 55-62. https://doi.org/10.1016/j.engfailanal.2012.02.004

[13] Cho, J.R., Lee, H.W., Jeong, W.B., Jeong, K.M. and Kim, K.W. (2013) Numerical Estimation of Rolling Resistance and Temperature Distribution of 3-D Periodic Patterned Tire. International Journal Solids and Structures, 50, 86-96. https://doi.org/10.1016/j.ijsolstr.2012.09.004

[14] Baranowski, P. and Malachowski, J. (2011) Numerical Investigations of Terrain Vehicle Tire Subjected to Blast Wave. Journal of KONES Powertrain and Transport, 18, 23-30.

[15] Cha, D.W. and Jeong, H.Y. (2011) Development of a Mode III Fatigue Test Method and a Peel Test Method for Tire Specimens. International Journal of Fatigue, 33, 912-920. https://doi.org/10.1016/j.ijfatigue.2011.02.002

[16] Alkan, V., Karamihas, S.M. and Anlas, G. (2011) Finite Element Modelingof Static Tire Enveloping Characteristics. International Journal Automotive Technology, 12, 529-535. https://doi.org/10.1007/s12239-011-0062-4

[17] Kongo Konde, A., Rosu, I., Lebon, F., Brardo, O. and Devesa, B. (2013) On the Modeling of Aircraft Tire. Aerospace Science and Technology, 27, 67-75. https://doi.org/10.1016/j.ast.2012.06.008

[18] Guan, Y.J., Zhao, G.Q. and Cheng, G. (2011) 3-Dimensional Non-Linear FEM Modeling and Analysis of Steady-Rolling of Radial Tires. Journal of Reinforced 
Plastics and Composites, 30, 229-240. https://doi.org/10.1177/0731684410391516

[19] Bris, S.S., Ungureanu, N., Maican, E., Murad, E. and Vladut, V. (2011) FEM Model to Study the Influence of Tire Pressure on Agricultural Tractor Wheel Deformations. Proceedings of International Scientific Conference Engineering for Rural Development, Bucharest, 26-27 May 2011, 223-228.

[20] Pidaparti, R.M.V. (1995) Hierarchical Bending Analysis of Cord-Rubber Composites. AIAA Journal, 33, 2359-2363. https://doi.org/10.2514/3.12992

[21] Pidaparti, R.M.V. (1996) Torsional Analysis of a Steel Cord-Rubber Tire Belt Structure. Tire Science and Technology, 24, 339-348.

https://doi.org/10.2346/1.2137526 